\title{
Space-like Penguin Effects in $B_{c}$ Decays *
}

\author{
Dong-Sheng $\mathrm{Du}^{\mathrm{a}, \mathrm{b}}$, Zheng-Tao $\mathrm{Wei}^{\mathrm{b}}{ }^{\dagger}$

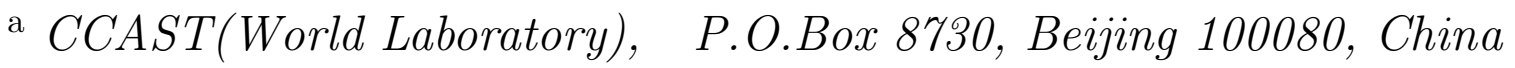 \\ b Institute of High Energy Physics, Chinese Academy of Sciences, \\ P.O.Box 918(4), Beijing, 100039, P. R. China $\ddagger$
}

\begin{abstract}
The space-like penguin contributions to branching ratios and CP asymmetries in charmless decays of $B_{c}$ to two pseudoscalar mesons are studied using the next-to-leading order low energy effective Hamiltonian and factorization approximation. Both the gluonic penguin and the electroweak penguin diagrams are considered. In addition the annihilation diagram contributions are also taken in account. We find that the space-like penguin effects are significant.
\end{abstract}

\footnotetext{
* Supported in part by National Natural Science Foundation of China

${ }^{\dagger}$ E-mail address: duds@bepc3.ihep.ac.cn; weizt@hptc5.ihep.ac.cn

${ }_{\ddagger}^{\ddagger}$ mailing address.
} 


\section{Introduction}

The weak decays of $B$ mesons offer a direct way to determine the Cabibbo-KabayashiMaskawa (CKM) matrix elements and to explore the origin of $\mathrm{CP}$ violation. Penguin diagrams can play an important role in charmless B decays. In most cases, attentions were paid to time-like penguin diagram in the literature, and the space-like penguin diagram is considered negligible because of the form factor suppression. In Ref.[1], space-like penguin diagram contribution to the branching ratios and $\mathrm{CP}$ violating asymmetries in $B_{u}^{-}, B^{0}$, $B_{s}^{0}$ decays are considered. The result shows that the space-like penguin amplitude can be enhanced by the hadronic matrix element involving $(\mathrm{V}-\mathrm{A})(\mathrm{V}+\mathrm{A})$ or $(\mathrm{S}-\mathrm{P})(\mathrm{S}+\mathrm{P})$ currents, and the space-like penguin effects are large in most charmless B decays.

$B_{c}$ meson is considered as the next and the last member of $\mathrm{B}$ mesons. Its physics has got intensive attention recently [2][3]. $B_{c}$ decay has its own characteristics. The obvious one is that $B_{c}$ carries $c$ and $b$ quarks, which are both heavy. So, $B_{c}$ decays can be interesting candidates for testing the spectator ansatz. We assume that spectator approximation can be applied in $B_{c}$ decays. In our paper, we will consider only $b$ quark decays and take $c$ quark as a spectator. From Ref.[3], the future accelerator, Large Hardron Collider (LHC), will produce $2.1 \times 10^{8} B_{c}$ per year, and can be a good place to study $B_{c}$ decays.

In this paper, we study space-like penguin diagram effects in $B_{c}$ decays to two pseudoscalars, and we concentrate on the charmless $B_{c}$ decays, because penguin diagram plays an important role in these decays. We use the next-to-leading order low energy effective Hamiltonian and factorization approxamation to calculate the branching ratios and CP violating asymmetries. In $B_{c}$ charmless decays, the annihilation diagram has the same order amplitude as the tree diagram. So the annihilation diagrams should be also taken into account. The result shows that the space-like penguin diagram contributions in $B_{c}$ 
charmless decays are large and can not be neglected.

\section{Effective Hamiltonian and Factorization Approxi-}

\section{mation}

We assume spactator approximation in $B_{c}$ decays: the $c$ quark is a spectator and the $b$ quark decays to other light quarks. According to reference [4], the next-to-leading order low energy effective Hamiltonian describing $|\Delta B|=1$ transitions is given at the renormalization scale $\mu=O\left(m_{b}\right)$ as

$$
\mathcal{H}_{e f f}(|\Delta B|=1)=\frac{G_{F}}{\sqrt{2}}\left[\sum_{q=u, c} v_{q}\left\{Q_{1}^{q} C_{1}(\mu)+Q_{2}^{q} C_{2}(\mu)+\sum_{k=3}^{10} Q_{k} C_{k}(\mu)\right\}\right]+H . C .
$$

The CKM factors $v_{q}$ are defined as

$$
v_{q}=\left\{\begin{array}{cl}
V_{q d}^{*} V_{q b} & \text { for } b \rightarrow d \text { transitions } \\
V_{q s}^{*} V_{q b} & \text { for } b \rightarrow s \text { transitions. }
\end{array}\right.
$$

The ten operators $Q_{1}^{u}, Q_{2}^{u}, Q_{3}, \ldots, Q_{10}$ are given as the following forms:

$$
\begin{array}{ll}
Q_{1}^{u}=\left(\bar{q}_{\alpha} u_{\beta}\right)_{V-A}\left(\bar{u}_{\beta} b_{\alpha}\right)_{V-A} & Q_{2}^{u}=(\bar{q} u)_{V-A}(\bar{u} b)_{V-A} \\
Q_{3(5)}=(\bar{q} b)_{V-A} \sum_{q^{\prime}}\left(\bar{q}^{\prime} q^{\prime}\right)_{V-A(V+A)} & Q_{4(6)}=\left(\bar{q}_{\alpha} b_{\beta}\right)_{V-A} \sum_{q^{\prime}}\left(\bar{q}_{\beta}^{\prime} q_{\alpha}^{\prime}\right)_{V-A(V+A)} \\
Q_{7(9)}=\frac{3}{2}(\bar{q} b)_{V-A} \sum_{q^{\prime}} e_{q^{\prime}}\left(\bar{q}^{\prime} q^{\prime}\right)_{V+A(V-A)} & Q_{8(10)}=\frac{3}{2}\left(\bar{q}_{\alpha} b_{\beta}\right)_{V-A} \sum_{q^{\prime}} e_{q^{\prime}}\left(\bar{q}_{\beta}^{\prime} q_{\alpha}^{\prime}\right)_{V+A(V-A)}
\end{array}
$$

where $Q_{1}^{u}$ and $Q_{2}^{u}$ are the current-current operators, and the current-current operators $Q_{1}^{c}$ and $Q_{2}^{c}$ can be obtained from $Q_{1}^{u}$ and $Q_{2}^{u}$ through the substitution of $u \rightarrow c . Q_{3}, \ldots, Q_{6}$ are the QCD penguin operators, whereas $Q_{7}, \ldots, Q_{10}$ are the electroweak penguin operators. The quark $q=d$ or $s$ for $b \rightarrow d$ or $s$ transitions, respectively; the indices $\alpha, \beta$ are $S U(3)_{c}$ color indices; $(V \pm A)$ refer to $\gamma_{\mu}\left(1 \pm \gamma_{5}\right)$. 
It is useful to use the renormalization scheme independent Wilson coefficient functions $[5]:$

$$
\overline{\mathbf{C}}(\mu)=\left[\hat{1}+\frac{\alpha_{s}(\mu)}{4 \pi} \hat{r}_{s}^{T}+\frac{\alpha(\mu)}{4 \pi} \hat{r}_{e}^{T}\right] \cdot \mathbf{C}(\mu),
$$

where $\mathbf{C}(\mu), \overline{\mathbf{C}}(\mu)$ are all column vectors. The matrix elements are:

$$
<\mathbf{Q}^{T}(\mu) \cdot \mathbf{C}(\mu)>\equiv<\mathbf{Q}^{T}>_{0} \cdot \mathbf{C}^{\prime}(\mu)
$$

where $<\mathbf{Q}>_{0}$ denote the tree level matrix elements of these operators, and $\mathbf{C}^{\prime}(\mu)$ are defined as

$$
\begin{array}{ll}
C_{1}^{\prime}=\bar{C}_{1}, & C_{2}^{\prime}=\bar{C}_{2}, \quad C_{3}^{\prime}=\bar{C}_{3}-P_{s} / 3, \quad C_{4}^{\prime}=\bar{C}_{4}+P_{s}, \\
C_{5}^{\prime}=\bar{C}_{5}-P_{s} / 3, & C_{6}^{\prime}=\bar{C}_{6}+P_{s}, \quad C_{7}^{\prime}=\bar{C}_{7}+P_{e}, \quad C_{8}^{\prime}=\bar{C}_{8}, \\
C_{9}^{\prime}=\bar{C}_{9}+P_{e}, & C_{10}^{\prime}=\bar{C}_{10},
\end{array}
$$

where $P_{s, e}$ are given by

$$
\begin{aligned}
P_{s} & =\frac{\alpha_{s}}{8 \pi} \bar{C}_{2}(\mu)\left[\frac{10}{9}-G\left(m_{q}, q, \mu\right)\right] \\
P_{e} & =\frac{\alpha_{e m}}{9 \pi}\left(3 \bar{C}_{1}+\bar{C}_{2}(\mu)\right)\left[\frac{10}{9}-G\left(m_{q}, q, \mu\right)\right] \\
G(m, q, \mu) & =-4 \int_{0}^{1} d x x(1-x) \ln \left[\frac{m^{2}-x(1-x) q^{2}}{\mu^{2}}\right],
\end{aligned}
$$

here $q=u, c$. The numerical values of the renormalization scheme independent Wilson Coefficients $\bar{C}_{i}(\mu)$ at $\mu=O\left(m_{b}\right)$ are $[6]$

$$
\begin{array}{llll}
\bar{c}_{1}=-0.313, & \bar{c}_{2}=1.150, & \bar{c}_{3}=0.017, & \bar{c}_{4}=-0.037, \\
\bar{c}_{5}=0.010, & \bar{c}_{6}=-0.046, & \bar{c}_{7}=-0.001 \cdot \alpha_{e m}, & \\
\bar{c}_{8}=0.049 \cdot \alpha_{e m}, & \bar{c}_{9}=-1.321 \cdot \alpha_{e m}, & \bar{c}_{10}=0.267 \cdot \alpha_{e m} .
\end{array}
$$

In Equation (7), $q^{2}$ denotes the momentum transfer squared of the virtual gluons, photons, and $Z^{0}$ appearing in the QCD and electroweak penguin didagrams respectively. So, the Wilson coefficients $C_{i}^{\prime}$ depend on $q^{2}$. We adopt a simple kinematic picture [1] for two body decays $B \rightarrow P P^{\prime}$ as illustrated as in Fig 1 . 
The average value of $q^{2}$ can be given by

$$
<q^{2}>=m_{b}^{2}+m_{q}^{2}-2 m_{b} E_{q}
$$

where $E_{q}$ is determined from

$$
E_{q}+\sqrt{E_{q}^{2}-m_{q}^{2}+m_{q^{\prime}}^{2}}+\sqrt{4\left(E_{q}^{2}-m_{q}^{2}\right)+m_{q^{\prime}}^{2}}=m_{b}
$$

for the time-like penguin diagram; and

$$
E_{q}+\sqrt{E_{q}^{2}-m_{q}^{2}+m_{q^{\prime}}^{2}}+=m_{b}+m_{q^{\prime}}
$$

for the space-like penguin diagram.

In exclusive nonleptonic decays, the current-current operator matrix element can be calculated by factorization approximation and BSW method [7].

For the tree diagram of Fig.2a which correponds to $b \rightarrow q \overline{q^{\prime}} q^{\prime}$, the matrix element for four-quark operator is defined as:

$$
\begin{aligned}
M_{q_{1} q_{2} q}^{P P^{\prime}} & \equiv<P P^{\prime}\left|\left(\overline{q_{1}} q_{2}\right)_{V-A}(\bar{q} b)_{V-A}\right| B_{c}^{-}> \\
& =<P\left|\left(\overline{q_{1}} q_{2}\right)_{V-A}\right| 0><P^{\prime}\left|(\bar{q} b)_{V-A}\right| B_{c}^{-}> \\
& =-i f_{P}^{\overline{q_{1}} q 2} f_{+}^{B_{c} P^{\prime}}\left(M_{P}^{2}\right)\left(M_{B_{c}}^{2}-M_{P^{\prime}}^{2}-\frac{M_{B_{c}}-M_{P^{\prime}}}{M_{B_{c}}+M_{P^{\prime}}} M_{X}^{2}\right)
\end{aligned}
$$

where $f_{+}^{B_{c} P^{\prime}}\left(m_{P}^{2}\right)=\frac{f_{+}^{B_{c} P^{\prime}}(0)}{1-M_{P}^{2} /\left(M_{B_{c}}^{\text {pole }}\right)^{2}} \cdot f_{+}^{B_{c} P^{\prime}}(0)$ can be calculated in BSW model, and $M_{B_{c}}^{\text {pole }}=$ $6.30 \mathrm{GeV}$. For the time-like penguin diagram, this factorization method is applied to calculate the time-like penguin operator matrix element.

For the annihilation diagram of Fig.2b corresponds to $b \bar{c} \rightarrow q \bar{c}$, the matrix element is $[8]:$

$$
\begin{aligned}
S_{q c c}^{P P^{\prime}} & \equiv<P P^{\prime}\left|(\bar{q} c)_{V-A}(\bar{c} b)_{V-A}\right| B_{c}^{-}> \\
& =<P P^{\prime}\left|(\bar{q} c)_{V-A}\right| 0><0\left|\left(\bar{c}^{\prime} b\right)_{V-A}\right| B_{c}^{-}> \\
& =i f_{B_{c}} f_{+}^{a}\left(M_{B_{c}}^{2}\right)\left(M_{P}^{2}-M_{P^{\prime}}^{2}-\frac{M_{P}-M_{P^{\prime}}}{M_{P}+M_{P^{\prime}}} M_{B_{c}}^{2}\right)
\end{aligned}
$$


The matrix elements are computed at momentum transfer $q^{2}=M_{B_{c}}^{2}$. We take the asymp-

totic form factor $f_{+}^{a}\left(M_{B_{c}}^{2}\right)=i 16 \pi \alpha_{s} f_{B_{c}}^{2} / M_{B_{c}}^{2}[9]$. One point should be noted: for the annihilation diagram, $C_{1}^{\prime}=\bar{C}_{2}, C_{2}^{\prime}=\bar{C}_{1}$.

In $B_{c}$ decays, the annihilation diagram is enhanced by the CKM factor $v_{c}$. For the $b \rightarrow d$ process, $\left|\frac{v_{c}}{v_{u}}\right| \approx 3$; for $b \rightarrow s$ process, $\left|\frac{v_{c}}{v_{u}}\right| \approx 57$. So, the annihilation diagram should be taken into account in $B_{c}$ decays.

For the space-like penguin diagram, just like the annihilation diagram, its factorization method is the same as that of the annihilation diagram.

\section{Numerical Calculation}

The decay width for a $B_{c}$ meson at rest decaying into two pseudoscalars is

$$
\Gamma\left(B_{c} \rightarrow P P^{\prime}\right)=\frac{1}{8 \pi}\left|<P P^{\prime}\right| H_{e f f}\left|B_{c}>\right|^{2} \frac{|\vec{p}|}{M_{B_{c}}^{2}}
$$

where

$$
|\vec{p}|=\frac{\left[\left(M_{B_{c}}^{2}-\left(M_{P}+M_{P}^{\prime}\right)^{2}\right)\left(M_{B_{c}}^{2}-\left(M_{P}-M_{P}{ }^{\prime}\right)^{2}\right)\right]^{\frac{1}{2}}}{2 M_{B_{c}}}
$$

is the momentum of the pseudoscalar meson $P$ or $P^{\prime}$. The corresponding branching ratios are given by

$$
\operatorname{Br}\left(B_{c} \rightarrow P P^{\prime}\right)=\frac{\Gamma\left(B_{c} \rightarrow P P^{\prime}\right)}{\Gamma_{t o t}^{B_{c}}}
$$

In our numerical calculation, we take[10] $\Gamma_{\text {tot }}^{B_{c}}=1.32 \times 10^{-12} \mathrm{GeV}$,

The $B_{c}$ meson decay amplitude can be generally expressed as

$$
<P P^{\prime}\left|H_{e f f}\right| B_{c}^{-}>=\frac{G_{F}}{\sqrt{2}} \sum_{q=u, c} v_{q} F_{q} .
$$

where $q=u, c$, and $F_{q}$ including the tree and and annihilation and penguin amplitude. 
The CP-violating asymmetry can be given by

$$
\begin{aligned}
\mathcal{A}_{c p} & \equiv \frac{\Gamma\left(B_{c}^{-} \rightarrow P P^{\prime}\right)-\Gamma\left(B_{c}^{+} \rightarrow \bar{P} \bar{P}^{\prime}\right)}{\Gamma\left(B_{c}^{-} \rightarrow P P^{\prime}\right)+\Gamma\left(B_{c}^{+} \rightarrow \bar{P} \bar{P}^{\prime}\right)} \\
& =\frac{2 \operatorname{Im}\left(v_{u} v_{c}^{*}\right) \operatorname{Im}\left(F_{c} / F_{u}\right)}{\left|v_{u}\right|^{2}+\left|v_{c}\right|^{2}\left|F_{c} / F_{u}\right|^{2}+2 \operatorname{Re}\left(v_{u} v_{c}^{*}\right) \operatorname{Re}\left(F_{c} / F_{u}\right)}
\end{aligned}
$$

We take the decay $B_{c}^{-} \rightarrow \eta D^{-}$as an example to illustrate the calculation of branching ratio $B r$ and $\mathrm{CP}$ asymmetry $\mathcal{A}_{c p}$ including space-like penguin diagram.

$$
\begin{aligned}
<\eta D^{-}\left|H_{e f f}\right| B_{c}^{-}>= & \frac{G_{F}}{\sqrt{2}} \sum_{q=u, c} v_{q}\left[\left(a_{2} \delta_{u q}+a_{3}+a_{4}-a_{6}+a_{8}-a_{9} / 2-a_{10}+\right.\right. \\
& \left.\frac{2 M_{\eta}^{2}}{\left(m_{d}+m_{d}\right)\left(m_{b}-m_{d}\right)}\left(a_{5}-a_{7} / 2\right)\right) M_{d d d}^{\eta D^{-}}+ \\
& \left.\left(a_{2} \delta_{c q}+a_{3}+\frac{2 M_{B_{c}}^{2}}{\left(m_{d}-m_{c}\right)\left(m_{b}+m_{c}\right)}\left(a_{5}+a_{7}\right)+a_{9}\right) S_{d c c}^{\eta D^{-}}\right] .
\end{aligned}
$$

where $a_{k}$ is defined as

$$
\begin{aligned}
& a_{2 i-1} \equiv \frac{C_{2 i-1}^{\prime}}{3}+C_{2 i}^{\prime} \\
& a_{2 i} \equiv C_{2 i-1}^{\prime}+\frac{C_{2 i}^{\prime}}{3},(i=1,2,3,4,5)
\end{aligned}
$$

and

$$
\begin{aligned}
& M_{d d d}^{\eta D^{-}}=-i f_{\eta}^{\bar{d} d} f_{+}^{B_{c}^{-} D^{-}}\left(M_{\eta}^{2}\right)\left[\left(M_{B_{c}}^{2}-M_{D^{-}}^{2}\right)-\frac{M_{B_{c}}-M_{D^{-}}}{M_{B_{c}}+M_{D^{-}}} M_{\eta^{2}}\right] \\
& S_{d c c}^{\eta D^{-}}=\frac{i}{\sqrt{3}} f_{B_{c}} f_{+}^{a}\left(M_{B_{c}}^{2}\right)\left[\left(M_{\eta}^{2}-M_{D^{-}}^{2}\right)-\frac{M_{\eta}-M_{D^{-}}}{M_{\eta}+M_{D^{-}}} M_{B_{c}}^{2}\right]
\end{aligned}
$$

where $\frac{1}{\sqrt{3}}$ arises from $\eta=\frac{\bar{u} u+\bar{d} d-\bar{s} s}{\sqrt{3}}$. The $a_{2} \delta_{c q}$ term in Eq.(19) is the annihilation diagram contribution.

The numerical results of the space-like penguin contributions to the branching ratios and CP-violating asymmetries are given in Table 1 and 2. We calculate the branching ratios and $\mathrm{CP}$-violating asymmetries with the tree and annihilation and time-like penguin contributions for comparison. All the parameters such as meson decay constants, form factors and quark masses needed in our calculation are taken as $f_{\pi^{ \pm}}=0.13 \mathrm{GeV}, f_{K}=$ $0.160 G e V[11], f_{\pi^{0}}^{\bar{u} u}=-f_{\pi^{0}}^{\bar{d} d}=f_{\pi^{ \pm}} / \sqrt{2} . \quad f_{\eta}^{\bar{u} u}=f_{\eta}^{\bar{d} d}=-f_{\eta}^{\bar{s} s}=0.077 G e V, f_{\eta^{\prime}}^{\bar{u} u}=f_{\eta^{\prime}}^{\bar{d} d}=$ $f_{\eta^{\prime}}^{\bar{s} s} / 2=0.054 G e V[12], f_{B_{c}}=0.5 G e V[3], f_{+}^{B_{c}^{-} D^{-}}(0)=0.595, f_{+}^{B_{c}^{-} D_{s}^{-}}(0)=0.605, m_{u}=$ 
$0.005 \mathrm{GeV}, m_{d}=0.01 \mathrm{GeV}, m_{s}=0.2 \mathrm{GeV}, m_{c}=1.5 \mathrm{GeV}, m_{b}=4.5 \mathrm{GeV}, M_{B_{c}}=6.27 \mathrm{GeV}$. and the Wolfenstein parametrized CKM parameters are [13]: $\lambda=0.22, A=0.8, \eta=0.34$, $\rho=-0.12$.

\section{Conclusion and discussion}

From Table 1 and 2 we can see the following features:

(i) For most of the charmless decays, space-like penguin contributions to branching ratios are large. The corrections to the branching ratio and $\mathrm{CP}$ violating asymmetries are more than $100 \%$.

(ii) For space-like penguin in $B_{c}$ decays, the contributions of the electro-weak penguins are negligible.

(iii) The annihilation diagram contribution can not be negligible in $B_{c}$ decays.

The reason for the large space-like penguin effects can be explained as follows:

(i) When calculating the matrix elememt of $(V-A)(V+A)$ current $\left\langle P P^{\prime}\left|(\bar{q} b)_{V-A}(\bar{c} c)_{V+A}\right| B_{c}>\right.$, there will appear a factor $\frac{2 m_{B_{c}}^{2}}{\left(m_{q}-m_{c}\right)\left(m_{b}+m_{c}\right)}$, this factor will enhance the space-like penguin effects.

(ii) The form factor $f_{+}^{a}\left(m_{B_{c}}^{2}\right)$ is not a suppression factor as usually considered. In $B_{c}$ decays, $f_{+}^{a}\left(m_{B_{c}}^{2}\right)=0.077$, so combine with $f_{B_{c}}=0.5$, the annihilation or space-like penguin matrix element $S^{P P^{\prime}}$ is nearly as that of the tree and time-like penguin matrix element $M^{P P^{\prime}}$.

(iii) The quark mass is an important and sensitive parameter. In our calculation, we have used the current mass. The values of quark mass will have direct effect on the penguin amplitude. Other effects, such as nonfactorization effect, final state interation can provide many uncertainties. 


\section{Acknoledgement}

This work is supported in part by National Natural Science Foundation of China and the Grant of State Commission of Science and Technology of China.

\section{References}

[1] D.S.Du, Z.Z. Xing, Phys.Lett.B 349 (1995) 215.

D.S.Du, M.Z.Yang, D.Z.Zhang, Phys.Rev.D 53 (1996) 249.

[2] D.S.Du, Z.Wang, Phys.Rev.D 39 (1989) 1342;

K.Cheung and T.C.Yuan, Phys.Lett. B 325 (1994) 481.

[3] D.S.Du, X.L.Li, Y.D.Yang, Phys.Lett. B 380 (1996) 193.

[4] A.J.Buras, M.Jamin, M.E.Lautenbacher, P.H.Weisz, Nucl.Phys.B 400 (1993) 37;

A.J.Buras, M.Jamin, M.E.Lautenbacher, Nucl.Phys.B 400 (1993) 75; Nucl.Phys.B 408 (1993) 209.

[5] A.Buras, M. Jamin, M.Lautenbacher, and P.Weisz, Nucl.Phys.B 370 (1992) 69; Nucl.Phys. B 375 (1992) 501.

[6] N. G. Deshpande and X. G. He, Phys. Lett. B336(1994) 471; Phys. Rev. Lett. 26 (1995) 74

N. G. Deshpande, X. G. He and J. Trampetic, Phys. Lett. B345 (1995) 547.

[7] M.Wirbel, B.Stech and M.Bauer, Z.Phys.C29(1985)637; Z.Phys.C34(1987)103.

[8] J.Bernabéu and C.Jarlskog, Z.Phys.C8, 233(1981);

Z.Z.Xing, Phy.Rev.D53(1996) 2847. 
[9] G.P.Lepage and S.J.Brodsky, Phys.Lett.B87(1979)359.

[10] Martin Beneke, Fermilab-Pub-95/401-T.

[11] Particle Data Group, M. Aguilar-Benitez et al., Phys. Rev. D50 (1994) 1173.

[12] H.Y.Cheng, B.Tseng, IP-ASTP-03-97, NTU-TH-97-08, September, 1997.

[13] A.Ali, D.London, Preprint CERN-TH 7248/94. 


\section{Figure Captions}

Fig. 1. Penguin diagrams for a $B_{c}$ meson decaying into two light pseudoscalar mesons $P$ and $P^{\prime}$ a) the time-like penguin diagram; b) the space-like penguin diagram. The subscripts "v" denote "vacuum". The dark dot stands for the contraction of the W-loop.

Fig. 2. The Tree diagram and the Annihilation diagram in $B_{c}$ decays. a) the Tree diagram. b) the Annihilation diagram. 


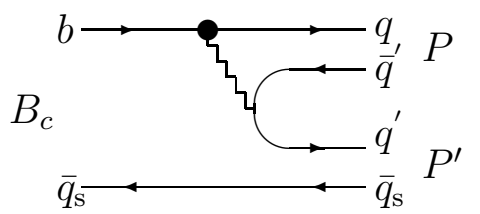

a)

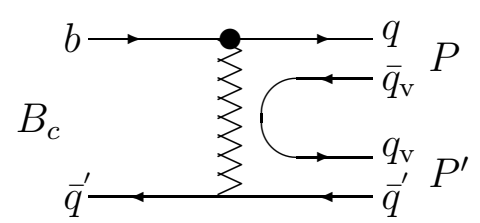

b)

Fig. 1.

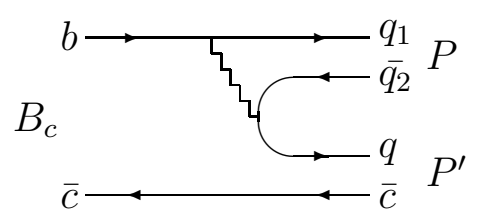

a)

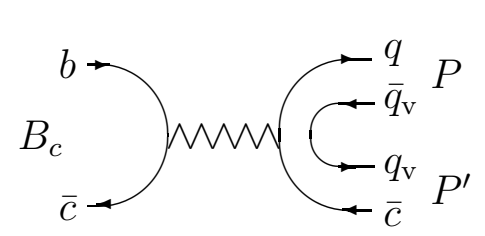

b)

Fig. 2 .

\section{Table Captions}

Table 1. The Branching Ratios of $B_{c}$ decaying to two pseudoscalar.

Table 2. The CP Asymmetries of $B_{c}$ decaying to two pseudoscalar. where the "Tree" means the tree diagram contribution, "Anni" means the annihilation diagram contribution, "T-like" denotes the time-like penguin contributions, the "S-like" denotes the space-like penguin contributions, "QCD" means QCD penguin contributions, and "EW" means electro-weak penguin contributions. 
Table 1

\begin{tabular}{|c|c|c|c|c|c|c|}
\hline \multirow{2}{*}{ Decay Mode } & \multicolumn{5}{|c|}{ Br } \\
\cline { 2 - 6 } & Only Tree & \multirow{2}{*}{ Tree+Anni } & \multicolumn{2}{|c|}{ Tree+Anni+T-like } & \multicolumn{2}{c|}{ Tree+Anni+T-like+S-like } \\
\cline { 4 - 7 } & & & QCD & QCD+EW & QCD & QCD+EW \\
\hline$B_{c}^{-} \rightarrow \pi^{-} \bar{D}^{0}$ & $1.12 \times 10^{-5}$ & $9.60 \times 10^{-6}$ & $8.31 \times 10^{-6}$ & $8.29 \times 10^{-6}$ & $2.27 \times 10^{-6}$ & $2.32 \times 10^{-6}$ \\
\hline$B_{c}^{-} \rightarrow K^{-} \bar{D}^{0}$ & $8.63 \times 10^{-7}$ & $2.81 \times 10^{-6}$ & $1.94 \times 10^{-5}$ & $1.99 \times 10^{-5}$ & $4.82 \times 10^{-5}$ & $4.76 \times 10^{-5}$ \\
\hline$B_{c}^{-} \rightarrow \pi^{0} D^{-}$ & $2.54 \times 10^{-8}$ & $1.20 \times 10^{-7}$ & $5.21 \times 10^{-7}$ & $3.73 \times 10^{-7}$ & $2.01 \times 10^{-5}$ & $1.91 \times 10^{-5}$ \\
\hline$B_{c}^{-} \rightarrow \eta D^{-}$ & $1.77 \times 10^{-8}$ & $3.00 \times 10^{-9}$ & $8.05 \times 10^{-6}$ & $7.73 \times 10^{-6}$ & $7.09 \times 10^{-6}$ & $7.10 \times 10^{-6}$ \\
\hline$B_{c}^{-} \rightarrow \eta^{\prime} D^{-}$ & $1.76 \times 10^{-8}$ & $9.88 \times 10^{-9}$ & $6.45 \times 10^{-5}$ & $6.43 \times 10^{-5}$ & $6.34 \times 10^{-5}$ & $6.34 \times 10^{-5}$ \\
\hline$B_{c}^{-} \rightarrow \eta D_{s}^{-}$ & $9.03 \times 10^{-10}$ & $1.53 \times 10^{-7}$ & $8.62 \times 10^{-6}$ & $2.71 \times 10^{-6}$ & $9.02 \times 10^{-6}$ & $8.87 \times 10^{-6}$ \\
\hline$B_{c}^{-} \rightarrow \eta^{\prime} D_{s}^{-}$ & $4.40 \times 10^{-10}$ & $1.26 \times 10^{-7}$ & $6.09 \times 10^{-6}$ & $9.18 \times 10^{-6}$ & $1.18 \times 10^{-5}$ & $1.17 \times 10^{-5}$ \\
\hline$B_{c}^{-} \rightarrow K^{0} D_{s}^{-}$ & 0 & $3.41 \times 10^{-8}$ & $1.29 \times 10^{-6}$ & $1.27 \times 10^{-6}$ & $1.99 \times 10^{-6}$ & $1.96 \times 10^{-5}$ \\
\hline$B_{c}^{-} \rightarrow \bar{K}^{0} D^{-}$ & 0 & $5.87 \times 10^{-7}$ & $1.60 \times 10^{-5}$ & $1.58 \times 10^{-5}$ & $3.60 \times 10^{-5}$ & $3.55 \times 10^{-5}$ \\
\hline
\end{tabular}

Table 2

\begin{tabular}{|c|c|c|c|c|c|}
\hline \multirow{2}{*}{ Decay Mode } & \multicolumn{5}{|c|}{$\mathcal{A}_{c p}$} \\
\cline { 2 - 6 } & \multirow{2}{*}{ Tree+Anni } & \multicolumn{2}{|c|}{ Tree+Anni+T-like } & \multicolumn{2}{c|}{ Tree+Anni+T-like+S-like } \\
\cline { 2 - 6 } & & QCD & QCD+EW & QCD & QCD+EW \\
\hline$B_{c}^{-} \rightarrow \pi^{-} \bar{D}^{0}$ & $-15.1 \%$ & $-6.8 \%$ & $-6.8 \%$ & $-80.6 \%$ & $-80.1 \%$ \\
\hline$B_{c}^{-} \rightarrow K^{-} \bar{D}^{0}$ & $92.6 \%$ & $-0.7 \%$ & $-0.7 \%$ & $19.7 \%$ & $19.7 \%$ \\
\hline$B_{c}^{-} \rightarrow \pi^{0} D^{-}$ & $92.7 \%$ & $27.9 \%$ & $34.1 \%$ & $15.1 \%$ & $15.1 \%$ \\
\hline$B_{c}^{-} \rightarrow \eta D^{-}$ & $-88.6 \%$ & $14.8 \%$ & $15.1 \%$ & $9.2 \%$ & $9.3 \%$ \\
\hline$B_{c}^{-} \rightarrow \eta^{\prime} D^{-}$ & $-47.7 \%$ & $12.1 \%$ & $12.1 \%$ & $11.6 \%$ & $11.6 \%$ \\
\hline$B_{c}^{-} \rightarrow \eta D_{s}^{-}$ & $-13.4 \%$ & $-0.7 \%$ & $-1.7 \%$ & $-1.4 \%$ & $-1.4 \%$ \\
\hline$B_{c}^{-} \rightarrow \eta^{\prime} D_{s}^{-}$ & $11.8 \%$ & $-1.8 \%$ & $-1.4 \%$ & $0.3 \%$ & $0.3 \%$ \\
\hline$B_{c}^{-} \rightarrow K^{0} D_{s}^{-}$ & 0 & $20.2 \%$ & $20.4 \%$ & $-0.6 \%$ & $-0.6 \%$ \\
\hline$B_{c}^{-} \rightarrow \bar{K}^{0} D^{-}$ & 0 & $-1.3 \%$ & $-1.3 \%$ & $0.1 \%$ & $0.1 \%$ \\
\hline
\end{tabular}

